\title{
MARIA THERESIA UND MARIA CHRISTINA ALS PROTOTYP UND VORBILD DER CHRISTLICHEN DAME FÜR UNGARISCHE ADELSFRAUEN (1765-1780) ${ }^{1}$
}

\author{
KATALIN CZIBULA \\ Eötvös Loránd Universität \\ czibula.katalin@gmail.com
}

\begin{abstract}
Der Text behandelt das Verhältnis von Maria Theresia zu ihren Kindern, allen voran der Erzherzogin Marie Christina, Gemahlin Alberts von Sachsen-Teschen, mit der sie eine innige Beziehung hatte. Beschrieben wird die mehrfach kodierte höfische Repräsentation der hohen Damen, die sich sowohl am Transport weltlicher als auch religiöser Werte manifestierte.
\end{abstract}

Schlüsselwörter: höfische Repräsentanz, adelige Frauen 18. Jhdt., Wiener Hof, Preßburger Hof, Religionsgeschichte, Religionspraxis, Ordensgeschichte

Trotz der Tendenz zur Säkularisierung in der zweiten Hälfte des 18. Jahrhunderts hielt sich auf dem Territorium des Habsburgerreiches ein sicherer und traditioneller Rahmen religiösen Lebens. So dominierte die Religiosität auch die Formen der Repräsentation auf dem Wiener Herrscherhof, wobei die Hauptstadt als Zentrum beispielgebend für das ganze Reich war.

Die religiöse Haltung der im Zentrum des höfischen Lebens stehenden Frauen boten ein Modell für die Frauen ihrer Epoche, und zwar sowohl in Bezug auf die Bekleidung als auch die Lebensart und das Wertesystem der Gesellschaft. Die höfischen Beispiele gestalteten das religiösische Leben der adeligen Frauen der Epoche, die „nach oben” blickten, das heißt ihre aufmerksamen Augen auf Wien richteten, wenn sie sich selbst ihrer Umwelt präsentieren wollten.

Die Formen der Wiener höfischen Repräsentation wurden ab der zweiten Hälfte des 18. Jahrhunderts nicht nur durch das barocke Vermächtnis, sondern auch immer mehr durch bürgerlichen Traditionen bestimmt. ${ }^{2}$ Maria Theresias Regierungszeit ist eine Epoche kulturgeschichtlicher Wandlung, in der - nach Habermas'schen Kategorien - die Foren und Institutionen der bürgerlichen Öffentlichkeit gegen die in der Barockzeit in der höfischen Repräsentation vorgegebenen Traditionen an Macht und Einfluss gewonnen haben. ${ }^{3}$

Die Wiener höfische Repräsentation sowie das religiöse Leben wurden nun definitiv dadurch charakterisiert, dass keine männliche sondern eine weibliche 
Persönlichkeit im Zentrum stand, die Repräsentationsangelegenheiten fokussierten somit auf eine Frauengestalt. Europa, im Gesamten betrachtet, kannte diese Tradition kaum, weil die Rolle des Repräsentierens üblicherweise von männlichen Herrschern besetzt war. Einzige Ausnahme ist der russische Königsthron, auf dem mehrere Zarinnen nach russischem Erbrecht saßen. ${ }^{4}$

Es steht die Frage im Raum, ob es neben den individuellen Eigenschaften des Herrschers gemeinsame Merkmale in den festgelegten Formen der Repräsentation gab. Tatsächlich lässt sich mit deren Hilfe auch die persönliche Note der Herrscherpersönlichkeit analysieren. Bereits die positivistische Geschichtsschreibung beforschte mit Vorliebe die Quellen zum Alltagslebens historischer Persönlichkeiten. Im späteren 20. Jahrhundert ${ }^{5}$ widmete man sich neben deren Alltag auch der Mentalität. Frühe Biographen Maria Theresias unterschieden zwischen der temperamentvollen, vitalen Lebensart der jungen Herrscherin und der frommen Lebensführung der verwitweten alten Kaiserin und sie vermerkten auch die Veränderungen, die mit diesem Prozess zusammenhingen.

\section{Maria Christina und Albert von Sachsen-Teschen}

Ab 1765 stand Ungarn vor einer speziellen Situation: Der Palatin Lajos Batthyány verstarb und Maria Theresia ernannte an seine Stelle keinen anderen Palatin, sondern erhob ihren Schwiegersohn, Herzog Albrecht/Albert Kasimir von Sachsen-Teschen, zum Statthalter von Ungarn und dadurch diente der Pressburger Hof, auf dem er und seine Frau lebten, als Statthalterzentrum. Das bedeutet aus unserer heutigen Sicht, dass innerhalb des Königreichs Ungarn ein Sekundärzentrum neben Wien eingerichtet wurde. Dieses Zentrum war nun auch als beispielgebend für die ungarischen Adeligen. Die zentrale Persönlichkeit an diesem Hof war zwar formell der Statthalter, Herzog Albrecht, tatsächlich spielte diese Rolle allerdings eine Frau, nämlich die Gattin des Statthalters, Erzherzogin Maria Christina - Maria Theresias Lieblingstochter.

Diese war als fünftes Kind der Kaiserin im Jahr 1742 als Maria Christina Johanna Josepha Antonia geboren worden. ${ }^{6}$ Maria Christina (auch: Marie Christine) wurde nach den strengen Regeln und Vorschriften des Hofes erzogen und von diesen geprägt. Ihre Erziehung verlief identisch mit jener der anderen Erzherzoginnen, doch entwickelte sie ein reges Interesse für die Künste. Sie erhielt Unterricht im Theaterspiel, trat in einigen Theaterstücken auf und musizierte nicht nur im Familienkreis, sondern auch vor Publikum. Das größte Talent zeigte sie in den bildenden Künsten. Mehrere erhaltene Zeichnungen und Gemälde weisen eine hohe Qualität auf, Maria Christina zeigte sich darin von den Merkmalen des bürgerlichen Rokoko inspiriert. ${ }^{7}$ Ihre Jugend war von zwei großen Erlebnissen geprägt: Zunächst war sie mit ihrer Schwägerin Isabella von Parma, der 
Frau des späteren Joseph II., in enger Freundschaft verbunden. Der Briefwechsel zwischen den zwei jungen Frauen ist voll Leidenschaft, sodass später immer wieder die Frage im Raum stand, ob dieser intime Ton in den Briefen nur die stilistisch-rhetorische Mode der Zeit widerspiegelte, oder doch ein Hinweis auf eine Liebesgeschichte sein könnte. Ein zweiter wichtiger Umstand, der sie von ihren Geschwistern unterscheidet, ist das innige Verhältnis zu ihrer Mutter. Zweifellos war nämlich sie das Lieblingskind. Dies lässt sich auch durch folgende Fakten nachvollziehen: zum einen symbolisch, und zwar dadurch, dass beide an einem 13. Mai geboren wurden und zum zweiten durch angeblich sehr ähnliche Charaktereigenschaften der beiden Frauen. Die Kaiserin/Königin zeigte bis zu ihrem Tod eine besonders innige Liebe und Achtung ihrer Tochter gegenüber.

Diese nicht zuletzt auch vereinnahmende Mutterliebe war charakteristisch für den Hof. „Felix Austria” hatte seine außenpolitischen Verbindungen jahrhundertlang auf gut überlegten Vermählungen aufgebaut, und diese Praxis wurde von Maria Theresia intensiv weiter verfolgt: durch das Stiften von Ehen, die die Braut, aber auch den Bräutigam gleichsam zu politischen Opfern werden ließen. ${ }^{8}$ Trotz dieser Heiratspolitik des Wiener Hofes konnte Maria Christina nach ihrer persönlichen Neigung heiraten, also quasi eine „Liebeshochzeit” schließen, allerdings musste die Kaiserin zunächst den gesellschaftlichen Status des künftigen Ehemannes signifikant anheben. So wurde der politisch bedeutungslose und minderbegüterte Herzog Albert von Sachsen-Teschen zum Ungarischen Statthalter ernannt. ${ }^{9}$ Er wurde im Jahr 1765 berufen, demselben Jahr, in dem Leopold, Großherzog der Toskana, heiratete. Während der Hochzeitsfeierlichkeiten in Innsbruck starb daselbst Kaiser Franz Stephan von Lothringen, Leopolds und Maria Christinas Vater, der eigentlich andere Pläne mit Maria Christina gehabt hatte als sie selbst. Aber der angehenden Verbindung stand nun nichts im Weg und das Paar heiratete sogar noch vor Ende des Trauerjahres, im April 1766.

Fortan ging vom Statthalterhof in Pressburg eine intensive Repräsentationstätigkeit aus. Die Kaiserin ließ die Pressburger Königsburg unter hohen Kosten renovieren. Berühmte Hof-Architekten aus Wien - Jean Nicolas Jadot de Ville-Issey, Nikolaus Pacassi und Franz Anton Hillebrandt - modernisierten das Gebäude. Das Mobiliar wurde aus Wien geholt, die Gemälde kamen aus der kaiserlichen Galerie, die Innenräume wurden vom Wiener Meister Maulbertsch, von Vinzenz Fischer und Josef Hauzinger dekoriert. ${ }^{10}$ Die Lieblingsvergnügung des Statthalterpaares war die Jagd, so bekam es von Maria Theresia das Jagdschloss und Landgut Halbthurn (Féltorony) in der Nähe von Pressburg zum Geschenk. ${ }^{11}$

Ein schriftlich erhaltenes Dokument der engen Mutter-Tochter Beziehung ist die lange Paränese, die Maria Theresia ihrer Tochter aus Anlass von deren Eheschließung und zum Beginn ihres eigenständigen Lebens schrieb. ${ }^{12}$ Dieser Brief zeigt eine ganz andere Tonart, anders als etwa die Intentionen der Kaiserin gegenüber ihrer anderen Tochter, der französischen Königin Marie Antoinette. 
Der Brief an Maria Christina zeigt eine ungewöhnliche Subjektivität und Freimütigkeit, beispielsweise, wenn die Mutter das Verhältnis zum späteren Ehemann gleichsam mit ihrem eigenen Lebenslauf vergleicht: „Deine Lage [ist] ebenso delikat, wie es die meinige war. Lasse ihn niemals Deine Ueberlegenheit (superiorité) fühlen. Man scheut keine Mühe, wenn man wahrhaft und vernünftig liebt; darüber bin ich ruhig." 13

Die Kaiserin wollte ihrer verheirateten Tochter zudem wichtige Ratschläge für den Umgang mit ungarischen Adeligen mit auf den Weg geben. Aus der Perspektive dieses Beitrags ist es hier wichtig, festzuhalten, dass sich dieser Text speziell mit Regeln des religiösen Lebens auseinandersetzt:

„An Sonntagen ist öffentlich Gottesdienst bei Hofe, Predigt, große Messe, um vier Uhr Vesper bei den Kapuzinern und Rosenkranz. Nur an Festtagen wie jenen der hl. Jungfrau, der Apostel oder an Landesfesten wirst Du Morgens öffentlich zur Kirche gehen, keine Predigt hören und immer die Kirche wechseln, um allen Geistlichen ein Vergnügen zu bereiten."14

Maria Theresia wies ihre Tochter zudem an, jeden Monat einen Kranken als Gast aufzunehmen und dazu alle religiösen Vertreter, Bischöfe, Prälaten und Domherren einzuladen. Auch diese Anweisungen zeigen, dass die Persönlichkeit Maria Theresias von starker Religiosität geprägt war, die sie auf ihre Tochter zu übertragen wünschte.

Über die Rolle und Gestaltung dieser Frömmigkeit am Pressburger Hof haben wir eine unmittelbare Quelle: Das erste Pressburger Periodikum, das genau zu dieser Zeit gegründet wurde, nämlich die Preßburger Zeitung. Die Nachrichten dieser Zeitung, die sich mit dem Hof beschäftigen, sind - so darf angenommen werden - verlässliche Dokumente von Augenzeugen.

Das Nachrichtenmaterial der Preßburger Zeitung stammte aus verschiedenen Quellen: Einerseits beschäftigte das Blatt eigene Korrespondenten und Journalisten aus dem Territorium des Königreichs, andererseits übernahm es auch weltpolitische und Reichsnachrichten - in erster Linie aus dem Wienerischen Diarium.

Über die Pressburger Begebenheiten berichtete die Zeitung mit großer Regelmäßigkeit, allerdings in wechselnder Ausführlichkeit: Zur Zeit des Landtags 1764 wurden umfangreiche Nachrichten vermeldet, später waren die Zeitungsberichte an bestimmte Begebenheiten gebunden. So wurde jedesmal über Besuche von Maria Theresia in der Stadt berichtet, besonders wichtig waren zudem die Ankünfte und Abreisen des Statthalterpaares, in relativer Kontinuität wurden die religiösen und weltlichen Ereignisse am pressburgischen Hof verewigt. Anhand der Berichterstattung der Presse kann man ein durch Religiosität geprägtes höfisches Leben rekonstruieren, das die Intimität des Rokoko der grandiosen Repräsentation des Barocks vorzog. Die durchaus bürgerlich geprägte Form der höfischen Vergnügungen fällt dabei ebenfalls auf. Im Zentrum der Repräsentation standen beide Frauen: die Kaiserin, die die Stadt häufig besuchte, und ihre an Kultur 
interessierte Tochter. Beide boten vorbildgebende Beispiele für die Leserschaft der Preßburger Zeitung, die alle höfischen Nachrichten im gesamten Königreich verbreitete und damit auch ein nicht zu unterschätzendes geschmacksbildendes Potential in sich trug.

Die Nachrichten in der Preßburger Zeitung geben auch Auskunft über das religiöse Leben und seine Praxis bei Hof zur Zeit der Statthalterei. In diesen Informationen erschienen Maria Theresia und Maria Christina als zentrale Persönlichkeiten, sie erlauben es uns auch, die Ordnung des religiösen Jahres am Pressburger Hof zu rekonstruieren. Das Statthalterpaar besuchte Wien zu bestimmten Anlässen und hielt sich wieder zu anderen in Pressburg auf. Aus diesen Aufenthalten lässt sich eine Jahres-Routine ableiten: So versammelte sich die Kaiserfamilie zu Neujahr immer in Wien und man besuchte gemeinsam die Neujahrsmesse in der Kirche der Augustiner. In der Zeit der Faschingsfestivitäten pendelten Maria Christina und Herzog Albert zwischen Wien und Pressburg hin und her. In dieser Saison standen weltliche Vergnügen wie Bälle und andere Lustbarkeiten im Vordergrund. Das Statthalterpaar brachte die Tradition der Schlittenfahrt aus Wien nach Pressburg, wo die dortigen Adeligen diesem Vergnügen auch in Abwesenheit des Paares nachgingen. Zur Karwoche kamen Maria Christina und Herzog Albert zwar immer nach Pressburg, sie verbrachten aber das Osterfest schon wieder in Wien. Nur ein einziges Mal, im Jahr 1769, gibt es eine Zeitungsnachricht, wonach Maria Christina und ihr Mann zu Ostern das Hochamt am 25. März im Pressburger Martinsdom besuchten. ${ }^{15}$ Das Fronleichnamsfest begingen sie in Pressburg, von dessen Prozessionen regelmäßig Berichte in der Zeitung erschienen. Der Sommer war zwar die Zeit von Reisen, doch die kaiserliche Familie feierte den gemeinsamen Geburtstag Maria Theresias und Maria Christinas am 13. Mai und jenen von Joseph II., am 13. März, immer in Wien. Schauplatz für die Advent- und Weihnachtszeit war für das Statthalterpaar immer Pressburg. Erst in den letzten Tagen des Jahres hielt man sich wieder in Wien auf.

Über sämtliche religiöse Feierlichkeiten erschienen oft sehr genaue Berichte in der Zeitung. ${ }^{16}$ So wurde zum Fronleichnamsfest nicht nur die Prozession sehr detailliert beschrieben, es ist vielmehr möglich, daraus die ganztägig stattfindenden Feierlichkeiten zu rekonstruieren: Morgens um 6 Uhr gingen die Handwerkszünfte mit ihren Fahnen von der Stadtpfarrkirche aus durch die Hauptstraße der Stadt, um 8 Uhr nahm das Statthalterpaar zusammen mit ihnen an der Heiligen Messe teil. Danach begann die Prozession: Zuerst kam eine große Menge des Volkes, es folgten die Leibdiener des Hofes und die Hausoffiziere, dann kamen der äußere und innere Stadtrat, die Kammerherren und die geheimen Räte, alle schwarz gekleidet, mit brennenden Fackeln in der Hand. Diesem Zug folgte der Bischof (von Jahr zu Jahr abwechselnd waren dies Sigmund Keglevich, der Bischof von Makarien, dann Georg Klimó, Bischof von Fünfkirchen oder Josef Batthyány, Bischof von Kalocsa). Der Prozessions-,,Himmel“ wurde von vier 
Kammerherren getragen, ihnen assistierten vier äußere Räte, gleich nach dem „Himmel“ folgte das Statthalterpaar mit dem Hofstaat, den Kavalieren und Hofdamen. Die Nachhut bildete eine Grenadierkompagnie der Schlossgarnison. Der Festzug hielt an vier Altären, die bei der Stadtmauer errichtet waren, um dort die Gottesdienste abzuhalten. Die Feier wurde von allerlei Toneffekten und Böllerschüssen begleitet. Ab den 70er-Jahren gesellten sich zur feierlichen Mittagstafel des Fronleichnamstages auch weltliche Unterhaltungen: So wurde im Jahr 1774 in der Sommerresidenz von Johann Erdődy eine Tafel für 22 Personen gedeckt und überdies wurden 12 Spieltische mit dem Montfaucon-Spiel aufgestellt. Bis $8 \mathrm{Uhr}$ abends dauerten die an das Mittagsmahl anschließenden Vergnügungen an. ${ }^{17}$ In den Jahren 1779 und 1780 besuchten der Statthalter und seine Gemahlin zudem auch Theateraufführungen (Der neureiche Ehemann, ${ }^{18}$ Die neubewohnte Insel oder die doppelte Versöhnung $\left.{ }^{19}\right)$.

Nach Fronleichnam verbrachten Maria Christina und Herzog Albrecht nur selten weitere religiöse Feste in Pressburg. Selbst den Tag des Heiligen Stephan nach Weihnachten feierten sie schon wieder in Wien. Der einzige Feiertag außer Fronleichnam, zu dem das Statthalterpaar allerdings regelmäßig in Pressburg erschien, war der Tag der Heiligen Cäcilia. Die Cäcilienfeier ähnelte in ihrer Art den Advent- und Weihnachtsfeierlichkeiten: Ort des Festes war aber nicht die Burg, sondern meistens die Domkirche von St. Martin. Den Höhepunkt des Ereignisses bildete ein Konzert, das die der Heiligen Cäcilia zugeschriebenen Eigenschaften unterstrich. Es traten professionelle Sängerinnen auf, wie das Fräulein Christine Frey von Schönstein und Kögl von Waldinutzy ${ }^{20}$ oder Fräulein Himmelbauer, eine Künstlerin, die mehrmals in der Stadt aufgetreten war. ${ }^{21}$ Die Berichterstatter erwähnten bei ihren Beschreibungen von Festlichkeiten zuweilen auch die Namen der Musiker: z.B. im Jahr 1773, am Tag der Heiligen Katharina, den Cellisten Himmelpauer (wahrscheinlich der Vater der ebenfalls genannten Sängerin $)^{22}$, einen Waldhorn-Künstler namens Kohl, ${ }^{23}$ im Jahr 1777, zur Feier der Heiligen Cäcilia, Anton Zistler, den Violinvirtuosen, ${ }^{24} 1779$ wurden außer Fräulein Himmelpauer noch Anton Zimmermann, Kapellmeister, und Xaverius Hammer, Violoncellist, erwähnt. ${ }^{25}$

Neben den kirchlichen Feierlichkeiten besuchten Kaiserin und Tochter Mönchsund Schwesternorden und deren Schulen zu Repräsentationszwecken. Diese zugleich auch karitativen Visiten fanden vor unterschiedlichem Publikum und an diversen Schauplätzen statt: Vor kleinerem Publikum, wie Orden oder Schulen, oder auch vor einer größeren Gesellschaft, den Einwohnern der Stadt, oder Mitgliedern des Hofes. In jedem Fall handelte es sich um die Präsentation höfischer Macht, und durch die dabei oft getätigten Spenden auch um die Demonstration von Freigiebigkeit: Die königlichen und kaiserlichen Majestäten beschenkten die besuchten Institutionen meist reich. Doch allein schon der hohe Besuch konnte das Prestige der jeweiligen Stätte und Gesellschaft steigern. 
Maria Theresia besuchte Pressburg durchaus häufig, was die Zeitung stets ausgiebig dokumentierte. Ihr hohes Ansehen sowie die Aufmerksamkeit, die ihr allseits geschenkt wurde, diente selbst in ihrer Abwesenheit noch der Imagepflege auch ihrer Tochter. Im Zentrum der Besuche bei Orden und Schulen standen jedenfalls immer beide Frauen, Maria Theresia und Maria Christina, denn diese Tätigkeit gehörte typischerweise zur ,weiblichen Repräsentation“. Hochrangige Männer fehlten meist bei diesen Gelegenheiten, und wenn sie doch erschienen, interessierten sie sich für andere Sehenswürdigkeiten.

Die von der kaiserlichen Familie präferierte Schule in Pressburg war das Kloster der Chorfrauen de Notre-Dame. Diese Frauenschule war von Maria Theresia für französisch sprechende hochadelige junge Frauen gegründet worden. Fast jedes Jahr besuchte sie die Zöglinge. Am 15. September 1774 begleiteten sie sogar der türkische Gesandte und dessen Sekretär, sowie der Hofdolmetscher Karl Edler von Bihn bei ihrem Besuch in der Klosterschule. Sie alle nahmen an einer Feier teil und bewunderten, so die Pressburger Zeitung, dabei das Tanztalent der Zöglinge. ${ }^{26}$

Während des Landtags von 1764 waren die Frauen der kaiserlichen Familie regelmäßige Gäste im Kloster der Chorfrauen de Notre-Dame: am 11. Juli besuchten sie ein Lustspiel im Kloster ${ }^{27}$ am 20. Juli sahen sich die Erzherzoginnen Maria Elisabeth, Maria Amalia und Herzogin Charlotte, Schwester von Kaiser Franz Stephan von Lothringen, Pietro Metastasios Melodram Ciro riconosciuto in französischer Übersetzung und Marc-Antoine Legrands Ballett Les Amazones modernes an. ${ }^{28} \mathrm{Am} 24$. August nahmen die Erzherzoginnen Maria Anna und Maria Christina an einer Theateraufführung teil, wo Metastasios La clemenza di Tito und Charles-Simon Favarts Ninette à la Cour ou Le caprice amoureux (eine „petite Bourlesque“) aufgeführt wurden. ${ }^{29}$ Am 27. September besuchte die Kaiserin die Schule erneut, am 28. verließ sie die Stadt wieder. ${ }^{30}$

Eine weitere feierliche Begebenheit war die Ankunft der kaiserlichen Familie nach der Hochzeit von Maria Christina und Herzog Albert: Maria Theresia, Joseph II. und der sächsische Herzog Clemens, der Bruder Herzog Alberts in Begleitung der Hofdame Gräfin von Goes, die Gräfinnen Balassa und Csáky besuchten die Congregation de Notre-Dame. Die Schwestern und die Zöglinge, die in Uniform gekleidet waren, standen vom Tor bis zu den Treppen Spalier. Maria Theresia erlaubte ihre Hand zu küssen, und dann zog sich die Kaiserin mit der Oberin des Klosters zurück. Inzwischen verbrachten die Männer die Zeit ganz anders: Der Kaiser zeigte Herzog Clemens das Gebäude, der seine Bewunderung dafür ausdrückte. Beide verließen das Kloster vor 7 Uhr, die Kaiserin blieb noch eine halbe Stunde länger. ${ }^{31}$

Neben der Congregation de Notre Dame spielten auch die Ursulinen eine überragende Rolle in der höfischen Repräsentation. Am 15. Dezember 1777 besuchten Maria Christina und die Erzherzoginnen das Kloster der Ursulinen, wo die 
Zöglinge ihre Prüfungen vor den Majestäten mit gutem Erfolg ablegten. Ein anderes Mal schenkte die Statthalterin den Zöglingen ein Glückstopf-Spiel, an dem auch Erzbischof Joseph von Batthyány teilnahm: er lieferte die Musik. ${ }^{32}$

Die weiteren Schwesternorden in der Stadt, die Klarissen, und die Schwestern des Hl. Elisabeth-Ordens wurden von den Mitgliedern der kaiserlichen Familie ebenfalls besucht, sie verbrachten allerdings vermutlich nicht viel Zeit bei ihnen und so berichtete auch die Zeitung kaum darüber.

Das Statthalterpaar nahm 1781 Abschied von der Stadt, um nun in den Niederlanden seinen Pflichten nachzugehen. Maria Christina verabschiedete sich persönlich von zwei Klöstern, der Congregation de Notre-Dame und den Ursulinen. ${ }^{33}$

Zum religiösen Leben des Statthalterpaares gehört auch ein wichtiges, für beide Personen gänzlich neues Ereignis: 1775 brachen Maria Christina und Herzog Albert auf die größte Reise ihres bisherigen Lebens auf. Das Ziel dieser ausgedehnten Reise, die von Dezember 1775 bis Juli 1776 dauern sollte, waren Besuche bei vier von Maria Christinas Geschwistern: in Parma, bei Erzherzogin Maria Amalia, der Gemahlin Herzog Ferdinands, dann in Florenz, bei Leopold, Großherzog der Toscana (und späterer Kaiser Leopold II.), sodann in Mailand, bei Erzherzog Ferdinand Karl, Gouverneur der Lombardei, und schließlich auch in Neapel, bei Maria Karolina von Österreich, Königin von Neapel und Sizilien. Neben ihren Aufenthalten in diesen Städten verbrachte das Paar auch längere Zeit in Rom und besichtigte die Sehenswürdigkeiten der Stadt. Das große Interesse für die Künste war ein zentraler Beweggrund für die gesamte italienische Reise gewesen.

Sein Aufenthalt in Rom zur Osterzeit samt einer Audienz beim Papst waren wichtige Ereignisse für das religiöse Leben des Statthalterpaares und für die Festigung seines katholischen Glaubens. Die Informationen, die die Preßburger Zeitung darüber brachte, stammten aber bereits aus zweiter Hand. Sie waren von ausländischen Journalen übernommen worden, lediglich die Selektion der Nachrichten weist auf die Subjektivität des ungarischen Redakteurs hin. Diese Reise-Nachrichten erschienen zwar regelmäßig, sie waren aber kürzer als die Berichte aus Ungarn. ${ }^{34}$ So informierte die Pressburger Zeitung ihre Leserschaft über die Ankunft des Statthalterpaares in Rom, wo es am Palmsonntag die Messe in der Sixtinischen-Kapelle gehört hatte. Vermeldet wird, dass der Heilige Vater ihnen danach genau jenen Palmenzweig zum Geschenk machte, den er während der Palmprozession in seiner Hand gehalten hatte. ${ }^{35}$ Die Erzherzogin und der Herzog verbrachten dem Bericht zufolge die Nacht in einem Raum bei der Reliquie des Heiligen Kreuzes, am 6. April hörten sie ein Stabat Mater mit der Musik "von Joseph Magherini". ${ }^{36}$ Die Ostermesse besuchten sie im Petersdom, und nahmen im Anschluss daran an der Audienz des Papstes teil. Am darauffolgenden Tag schenkte ihnen der Hofmeister des Papstes, der Theologe Monsignore Giovan- 
ni Andrea Avogadro, mehrere wertvolle Kunstwerke. Schließlich reiste das Paar nach Neapel ab. ${ }^{37}$

Der kurze Rom-Bericht in der Zeitung konzentriert sich auf die religiös relevanten Begebenheiten: Erwähnt sind drei Schauplätze, an denen sich die beiden aufgehalten hatten, nämlich die Sixtinische Kapelle, die Anwesenheit beim Heiligen Kreuz sowie der Dom von St. Peter. Außer dem Papst werden aber doch zwei Persönlichkeiten namentlich erwähnt, die sich nicht durch religiöse, sondern kulturell-künstlerische Tätigkeiten auszeichneten: Es sind dies ein Komponist namens Magherini und ein Gelehrter namens Monsignore Avogadro. Der Redakteur hebt auch den Palmzweig hervor, der vom Papst getragen und geweiht worden ist, somit kein künstlerisches Produkt, sondern ein sakrales Objekt, das vom Papst berührt worden war und das eine wichtige Rolle im Palmsonntagsritual spielt. Dieser Zweig bildet die Ausnahme im sonst von der Reise Mitgebrachten, denn das Statthalterpaar hatte in deren Verlauf viele Kunstwerke und Gemälde gesammelt. Diese Erwerbungen waren für die Erzherzogin und den Herzog von so großer Bedeutung, dass sie den Maler Friedrich Heinrich Füger die Szene auf einem Gemälde verewigen ließen, in der sie Maria Theresia die in Italien erstandenen Kunstwerke zeigen. ${ }^{38}$

Maria Christina hatte also zwei Seiten: jene der kunstsinnigen Sammlerin und gleichzeitig die einer religiösen Frau, die auch die Rolle, die ihre Mutter vorlebte, perfekt zu tragen wusste.

In den 15 Jahren, in denen Pressburg als Statthalterhof fungierte, baute Maria Theresia ihr Erscheinungsbild als verwitwete, christlich tiefreligiöse Kaiserin sukzessive aus. Ihre Tochter Maria Christina folgte diesem Beispiel und hielt sich sehr genau an die ihr von ihrer Mutter gegebenen Anweisungen. Die Beispielwirkung dieser beiden Frauen bot schließlich auch den Rahmen für das religiöse Leben der ungarischen adeligen Frauen, die in einem breiten geographischen Radius ihre Informationen nicht zuletzt aus der Preßburger Zeitung bezogen.

\section{Anmerkungen}

1 Die Forschung wird durch das Projekt des Ungarischen Forschungsfond NKFI 119865 unterstützt.

2 In diesem Aspekt thematisierte die Ausstellung und deren Katalog anlässlich des 200. Jubiläums des Todes von Maria Theresia und dem Ende ihrer Regierung: Gerda Mraz und Gerald Schlag (Hrsg.): Maria Theresia als Königin von Ungarn: Ausstellung: Schloss Halbturn, 15. Mai-26. Oktober 1980. veranst. von der Kulturabteilung des Amtes der Burgenländischen Landesregierung (Eisenstadt: Rötzer Druck, 1980), später Kerstin Schmal: Die Pietas Maria Theresias im Spannungfeld von Barock und Aufklärung (Frankfurt am Main: Lang, 2001), Franz Herre: Maria Theresia, die große Habsburgerin (München: Piper, 2004) und Werner Telesko: Maria Theresia (Wien: Böhlau, 2012) 
3 Diese Charakteristika der Epoche wurde schon vor ca. 30 Jahren von Géza Galavics betont. Siehe: Galavics, Géza: Barockkunst, Repräsentation in Ungarn - In: Gerda Mraz (Hrsg.): Maria Theresia als Königin von Ungarn. Eisenstadt, 1984 (=Jahrbuch für österreichische Kulturgeschichte X.) 60-61.

4 Allerdings erschien ein Buch mit den beiden Lebensbeschreibungen von Maria Theresia und Zarin Katharina II. Hinsichtlich Charakter und Lebensführung der zwei Herscherinnen werden mehrere Parallelen angezeigt, doch betont das Buch die Unterschiede, jedoch nie die Ähnlichkeiten der beiden Frauen: Alekszandr Kamenszkij: Nagy Katalin, und Niederhauser, Emil: Mária Terézia, (Budapest: Pannonica, 2000). Das 300-jährige Jubiläum der Geburt der Kaiserin gab der Bewertung ihrer Regierungszeit neuen Schwung. Siehe: Thomas Lau: Die Kaiserin. Maria Theresia (Wien - Köln - Weimar, Böhlau, 2016). digitalisiert: https://books. google.hu/books? $\mathrm{id}=\mathrm{i} 5 \mathrm{EwDwAAQBAJ} \&$ printsec $=$ frontcover $\& \mathrm{hl}=\mathrm{de} \# \mathrm{v}=$ onepage $\& \mathrm{q} \& \mathrm{f}=$ false (2018.08.03), Michaela Pfundner - Gabriele Mauthe (Hrsg.): Maria Theresia: Habsburgs mächtigste Frau (Wien: Metroverlag, 2017), Elfriede Iby - Martin Mutschlechner - Werner Telesko - Karl Vocelka: Maria Theresia 1717-1780: Strategin - Mutter - Reformerin. (Wien, Amalthea, 2017) und Günther Haller - Rainer Nowak (Hrsg.): Maria Theresia Österreichs große Herrscherin 1717-1780; die unvorbereitete Thronerbin; die Ehefrau, Mutter und Landesmutter; Schulreformen; die Monarchie wird modernisiert; Schönbrunn; Marie Antoinette; was von ihr blieb (Wien: „Die Presse”-Verlags-Ges.m.b.H. \& Co KG, 2017) Elisabeth Badinter: Die Macht der Frau (München: btb, 2018).

5 In letzten Jahren reflektiert die ungarische Alltagsforschung in erster Linie auf die Handbücher, auf die deutsche populärwissenschafliche Literatur und auf ihre Übersetzungen. Siehe: Brigitte Vacha -Walter Pohl - Karl Vocelka (Hrsg.): A Habsburgok: Egy európai dinasztia története (Budapest: Gulliver, 1992), Brigitte Hamann (Hrsg.): Habsburg Lexikon (Budapest: Új Géniusz, 1988), Friedrich Weissensteiner: Mária Terézia leányai, [Übers. Szántó, Judit], (Budapest: Fabula, 1995), Franz Herre: Mária Terézia [Übers. Kajtár, Mária], (Budapest: Magyar Könyvklub, 2001), Hannes Etzlstorfer: Mária Terézia a gyermekszobában: egy császárnö magánélete [Übers. Kajtár, Mária], (Budapest: Gabo, 2011). Die Briefwechsel der Familienmitglieder dienen als unerschöpfliche Quelle zur Erforschung des Privatlebens der Kaiserin und ihrer Familie: Hans Schlitter (Hrsg.): Briefe der Erzherzogin Marie, Statthalterin der Niederlande an Leopold II. nebst einer Einleitung zur Geschichte der französischen Politik Leopolds II. (Wien: Gerold, 1896) digitalisiert: https://archive.org/details/briefedererzher01schlgoog (2018.08.03.), Alfred von Arneth (Hrsg.): Briefe der Kaiserin Maria Theresia an ihre Kinder und Freunde (Wien: Braunmüller, 1881) digitalisiert: https://archive.org/details/briefederkaiser01thergoog (2018.08.03.) Maria Theresia: Familienbriefe, (Berlin: Ullstein \& Co., 1920), Paul Christoph (Hg.): Maria Theresia. Geheimer Briefwechsel mit Marie Antoinette, (Frankfurt/M.: Ullstein, 1991), Monika Czernin - Jean-Pierre Lavandier: Maria Theresia -- Liebet mich immer: Briefe an ihre engste Freundin (Wien: Ueberreuter, 2017).

6 Das Leben der Erzherzogin Maria Christina (auch: Marie Christine) wurde von Adam Wolf mit großer Präzision im 19. Jahrhundert erforscht: Adam Wolf: Marie Christine, Erzherzogin von Oesterreich, Bd. I-II. (Wien: Gerold, 1863), Adam Wolf: Leopold II. und Marie Christine: Ihr Briefwechsel (Wien: Gerold, 1867). Die Fachliteratur beschäftigt sich oft mit dem Verhältnis zwischen Mutter und Tochter: Friedrich Weissensteiner: Die Töchter Maria Theresias (Berlin: Bastei Lübbe, 1996), Marie Christine - In: Brigitte Hamann (Hg.): Die Habsburger, (Wien: Ueberreuter, 1988) 312-315, Brigitte Hamann: Maria Christine (Marie), Erzherzogin von Österreich - In: Neue Deutsche Biographie, Band 16, (Berlin: Duncker \& Humblot, 1990) 200.f, digitalisiert: http://www.deutsche-biographie.de/sfz58361.html (2018. 08. 03.) 
7 Ihr am öftesten publiziertes Gemälde, „Das Nikolausfest”, stellt die kaiserliche Familie in einem bürgerlichen Idyll im Stil des Biedermeier dar. Als talentreiche Porträtmalerin verfertigte Maria Christina auch Bildnisse ihrer Eltern und aller Geschwister an.

8 Ein oft zitiertes Beispiel dieser Ehepolitik ist der Fall dreier Töchter Maria Theresias mit dem König von Neapel, Ferdinand IV. Dieser war in ganz Europa bekannt für seinen problematischen Charakter und seine bacchantische Lebensführung. Trotzdem wurde er mit drei Erzherzoginnen des Wiener Hofs verlobt: Noch als Kind wurde die Erzherzogin Maria Johanna mit ihm verlobt, nach deren frühem Tod folgte Erzherzogin Maria Josepha. Doch diese verstarb wenige Wochen nach ihrer Heirat, worauf die dritte Erzherzogin, Maria Karolina, Frau des Königs von Neapel wurde. Siehe: ifj. Barta, János: Mária Terézia (Budapest: Gondolat, 1988.) 112.

9 Walter Koschatzky - Selma Krasa: Herzog Albert von Sachsen-Teschen 1738-1822. Reichsfeldmarschall und Kunstmäzen. Veröffentlichung der Albertina (Wien: Österreichischer Bundesverlag, 1982) und Christian Benedik: Die Albertina, das Palais und die habsburgischen Prunkräume (Wien: Brandstätter, 2008.)

10 Géza Galavics: Die künstlerische Repräsentation der Habsburger-Könige in Ungarn bis 1848 - In: István Fazekas - Gábor Ujváry (Hrsg.): Kaiser und König. Eine historische Reise: Österreich und Ungarn 1526-1918. (Wien: Collegium Hungaricum, 2001.) 15.

11 Ebenda

12 Die Paränese umfasst einen deutsch- und einen französischsprachigen Teil; ein Ausschnitt in Deutsch: Wolf: op.cit. Bd. I. 63-73., die Ratschläge über das Privatleben auf Französisch:. II. 215-220.

13 Wolf: op.cit. Bd. I. 69.

14 Wolf: op.cit. Bd. I. 63.

15 Preßburger Zeitung 29. März 1769. No. 25.

16 Preßburger Zeitung 31. Mai 1766. No. 44. Preßburger Zeitung 27. Mai 1769. No. 42. Preßburger Zeitung 12. Juni 1773. No. 47. Preßburger Zeitung 4. Juni 1774. No. 45. Preßburger Zeitung 17. Juni 1775. No. 48. Preßburger Zeitung 20. Juni 1778. No. 49. Preßburger Zeitung 5. Juni 1779. No. 45. Preßburger Zeitung 27. Mai 1780. No. 43.

17 Preßburger Zeitung 8. Juni 1774. No. 46.

18 Preßburger Zeitung 5. Juni 1779. No. 45.

19 Preßburger Zeitung 27. Mai 1780. No. 43.

20 Preßburger Zeitung 24. November 1773. No. 94.

21 Preßburger Zeitung 24. November 1779. No.94.

22 Robert Eitner: Biographisch-bibliographisches Quellen-Lexikon der Musiker und Musikgelehrten der christlichen Zeitrechnung bis zur Mitte des XIX. Jahrhunderts, 11 Bände. (Graz: Breitkopf, 1900-1960.) Bd. 5. 157.

23 Preßburger Zeitung 27. November 1773. No. 95.

24 Preßburger Zeitung 26. November 1777. No. 95.

25 Preßburger Zeitung 24. November 1779. No. 94.

26 Preßburger Zeitung 21. September 1774. No. 76.

27 Preßburger Zeitung 14. Juli 1764. No. 1. Siehe: Zoltán Baranyai: A francia nyelv és müveltség Magyarországon: XVIII. század, (Budapest: Pfeifer, 1920.) S. 84-91. und Márta Zsuzsanna Pintér : Francia nyelvü színielöadások Pozsonyban a 18. században. - In: Iván Gyurcsík- Izabella Jégh-Zsuzsanna Papp (Hg.): Pozsony - Preßburg - Bratislava. A 700 éves város. Die 700 Jahre alte Stadt. 700-rocné mesto (Pozsony-Budapest, 1994.) 122-128.

28 Preßburger Zeitung 21. Juli 1764. No. 3. 
29 Preßburger Zeitung 25. August 1764. No. 13. Anhang

30 Preßburger Zeitung 29. September 1764. No. 23.

31 Preßburger Zeitung 19. April 1766. No. 32.

32 Preßburger Zeitung 20. Dezember 1777. No. 102.

33 Preßburger Zeitung 3. Januar 1781. No. 1.

34 In den 70er-Jahren finden sich deutlich kürzere Nachrichten über den Statthalterhof.

35 Preßburger Zeitung 24. April,1776. No. 33.

36 Preßburger Zeitung 27. April 1776. No. 34.

37 Preßburger Zeitung 1. Mai 1776. No. 35.

38 Heute ist das Gemälde in der Sammlung der Galerie Belvedere Wien aufbewahrt. 